\title{
Silver Digital Patient, a New Emerging Stakeholder in Current Healthcare. ProActiveAgeing: A Case Study
}

\author{
Marilena IANCULESCU, Adriana ALEXANDRU \\ National Institute for Research and Development in Informatics, \\ 8-10 Averescu Avenue, 011455 Bucharest, Romania, \\ manina@ici.ro, adriana@ici.ro
}

\begin{abstract}
Global ageing has already started to have a strong impact on society and economy. However, most national governments as well as urban and rural populations have not been adequately prepared for that so far. Elderly people are one of the most important category of healthcare consumers due to the degenerative disorders. eHealth can offer tremendous opportunities and facilities for supporting older patients to receive the most appropriate care so that they may lead a healthy, active and dignified life. An increased number of ageing people have become aware of the benefits provided by digital technology. A silver digital patient is an older person that has the skills, knowledge, literacy and willingness to use eHealth tools and services for getting better informed and more involved in managing his/her healthcare. Older patient-centered digital technology must be implemented for a broader acceptance. ProActive Ageing project is an example of that; it has as outcome an integrated platform for online services that provides useful tools for empowering ageing persons to take an even more active role in personal care related decisions and for enabling healthcare specialists to provide instruments for specific strategies, protocols and methods aimed at maintaining an adequate level of control over biologic and social functions of ageing people.
\end{abstract}

Keywords: eHealth, silver digital patient, ageing, integrated platform for online services

\section{Introduction}

The age structure of the EU population is changing dramatically. Elderly people are projected to account for an increasing share of the population. Those aged 65 and over will become a much larger part (rising from $17 \%$ to $30 \%$ of the population), and those aged 80 and over (rising from $5 \%$ to $12 \%$ ) will almost become as numerous as the young population in 2060. An ageing population will have a strong upward impact on public spending for long-term care. This is because frailty and disability rise sharply at older ages, especially amongst the very old (aged $80+$ ) which will be the fastest growing segment of the population in the decades to come [10].

Specific anti-ageing medical, information and communication research and technology, healthier lifestyles have not just increased longevity but have also decreased the morbid years - when people lose their functional independence and their minds and bodies break down.

Active ageing is the process of optimizing opportunities for health, participation and security in order to enhance quality of life as people age [13]. Active ageing allows people to realize their potential for physical, social, and mental well-being throughout the life course and to participate in society, while providing them with adequate protection, security and care when they need.

Healthy ageing is the process of optimising opportunities for physical, social and mental health to enable older people to take an active part in society without discrimination and to enjoy an independent and good quality of life [6]. Healthy ageing is a broad concept including physical as well as mental health and social well-being and support the adoption and maintenance by individuals of attitudes and behaviours known to promote health and well-being. In order to help their patients to have a successful ageing, healthcare specialists should apply up-to-date good practices, strategies and research. An active ageing can't exist without the health component and for that, new and adapted tools, services and practices must be provided both to individuals and healthcare specialists.

Information and Communication Technology (ICT) is essential as a source of innovation, as it enables integrated person - centred care, with more focus on prevention, early detection and independent living, to be provided in his/her own work and home environment. It can empower people to manage their health and support their healthy lifestyle choices. Therefore the market share of eHealth is expected to grow significantly: 
- The global telemedicine tools market will likely reach $\$ 43.4$ billion within five years, according to a new report published by Wellesley, Massachusettsbased BCC Research;

- The market for telehome technologies, or tools that enable outpatients to receive telehealth services, is predicted by BCC to grow from $\$ 6.5$ billion in 2013 to $\$ 24$ billion by 2019 ;

- PWC forecasts that the global mobile health market will reach a value of $€ 17.5$ billion in 2017, with Europe being the largest market segment ( $€ 5.2$ billion);

- Berg Insight forecasts that the installed base of smart home systems in the EU will grow at a rate above $50 \%$ in the next five years to reach 17.4 million smart homes installed in by 2017 ;

- McKinsey Global Institute estimates the potential economic impact of the Internet of Things to be $\$ 2.7$ trillion to $\$ 6.2$ trillion per year by 2025. Across the healthcare applications, Internet of Things technology could have an economic impact of $\$ 1.1$ trillion to $\$ 2.5$ trillion per year by 2025 [1].

A digital patient aims to increase his/her knowledge, information and skills in the health domain in order to better manage his/her own health and care by using digital technologies. These have the huge potential to support the leveraging of the patient's empowerment and awareness of being directly involved in the healthcare process and to allow the healthcare services to be safer, more affordable, efficient and accessible.

Figure 1 synthesizes the main characteristics of a digital patient [7].

A silver digital patient can be defined as an older patient with an appropriate level of health and IT literacy that has become more comfortable using digital technology and services.

A silver digital patient is an elderly person that has surpassed some challenges in adopting new technologies like physical disorders, skeptical attitudes about the benefits of digital technology or difficulties in learning to use new digital devices.

The new waves of ageing population are more experienced with digital technology than the current older population and much more

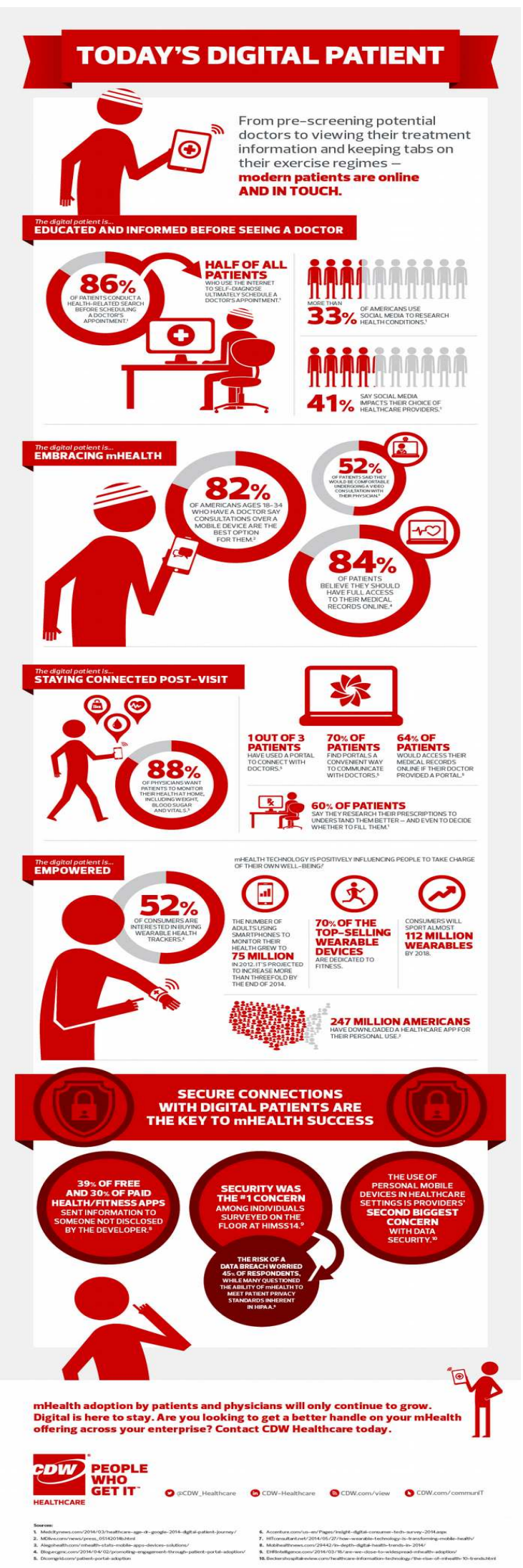

Figure 1. Digital patient's main characteristics

interested in being engaged in their care; therefore healthcare systems have to become more convenient for patients of all ages. 
Despite the growing number of elderly people that have accepted the use of digital technology as a routine in their lives, there is still an important group of older persons that are exposed to digital divide ("the economic, educational, and social inequalities between those who have computers and online access and those who do not" - Oxford Dictionary).

Bridging digital divide is a target healthcare and eHealth providers should better take into consideration in designing and implementing more appropriate digital technology.

In this context, this paper presents the ProActive Ageing project which is an integrated platform for online services that highlights the importance of applying new approaches, methods, means and researches for adopting an active, independent and healthy ageing. It focuses on developing online tools for:

- A sustainable (re)integration of ageing persons in social and working life;

- Enhancing the own health and independence of an ageing person, with a strong emphasis on lifelong learning, knowledge sharing;

- Providing comprehensive training courses addressed to formal carers (specialists) of ageing persons;

- Achieving more efficient methods of delivering improved outcomes from healthcare research, good practice and a better service to patients.

It facilitates the empowerment of the silver digital patients and ageing persons by providing tools for a more "person-centric" social and healthcare system in which individuals need to take an even more active role in personal care related decisions.

\section{Silver Digital Patient, Profile and Demands}

Demographic shift brings new challenges and opportunities for healthcare providers. Major adjustments have to be implemented such as reconsidering care options with a stronger accent on home-care or using new technologies able to play increasingly active role in medical services or self-healthcare.

The silver economy concept seeks to look holistically at ageing and the opportunities it presents, bearing on the future direction of a broad range of polices such as those on the built environment, 50+ employment, life-long learning and preventative healthcare. Moreover, it seeks to embrace new technologies (e.g. health monitoring, smart homes, and care robots) and use them to lower the costs of ageing and improve the lives of older citizens whilst simultaneously helping to boost the economy [4].

The end-user development has become the most commonly used form of programming. It includes customization, integration and extension activities which may lead to a significant alteration of the system and may raise serious issues concerning correctness and consistency, security, and privacy [12].

Designers have started to develop digital tools and technology having as a main core open-minded towards eHealth older patients, taking into consideration their specific needs and interests.

A silver digital patient is looking for more than a simple access to healthcare information. Due to the increasing integration of digital technology in daily activities, elderly become more and more interested in having online access to their personal healthcare information, to health specialists and furthermore, they expect to have eHealth tools able to support them in managing their own care.

Older patients prefer traditional digital channels such as websites and e-mail, while younger patients are, unsurprisingly, more open to newer channels such as social media. A report from the European Union suggests that service type should be segmented by age; younger patients, want access to health-promotion and prevention services, whereas older patients need information about services for acute and chronic conditions. But both groups seek information at the same rates [2].

In terms of overall technology use, 73 percent of older adults reported using cellular phones and 63 percent reported using computers, which has remained steady since 2011 . On the other hand, this survey showed that tablet use is increasing, jumping from under 20 percent to around 25 percent of older adults from 2013 to 2014 alone. As for online activities, 43 percent of older adults reported using e-mail or texts, an increase of approximately 3 percent since 2011. Social networking use is also increasing, 
with the percentage using these sites or apps at just below 20 percent. There were smaller increases in the percentage of older adults doing internet banking (approximately 20 percent) and online shopping (approximately 15 percent) [11].

In terms of digital health technology use, there were again increases from 2011 to 2014, although overall use remains low. Overall, "the proportion of seniors who used any digital health increased from $21 \%$ in 2011 to $25 \%$ in 2014." While 60 percent of the general population searches for health information online, only 18 percent of older adults reported doing this in 2014, an increase from 15.5 percent in 2011. The other health technology use asked about in this survey was used by even fewer older adults, with around 10 percent using technology to contact physicians and fill prescriptions, and under 5 percent using technology to handle insurance [11].

The common profile of a silver digital patient comprises the following main characteristics:

- Active user of digital interactive media and mobile communications;

- Having enhanced levels of health and computer literacy;

- Suffering of some degenerative disabilities (vision and hearing decline, cognition disorders, motor skills impairment etc.);

- Less confident than a younger patient in direct benefits of digital technology;

- Poorer adaptability for the change, including devices and their user-interfaces;

- More analytic in decision-making;

- More demanding to receive better, information and care services;

- Requiring longer learning times in using a new technology;

- Highly interested in receiving the best medical services in his own private environment for a prolonged independent living;

- Willingly to improve social participation interaction and activity;

- More likely to accept new technology that prove its usefulness, benefits and efficiency;

- Accepting the necessity of long-life education.

Ageing societies face tough challenges namely in terms of the pressure on their healthcare and social security systems, which makes it urgent to find new models to accommodate current demographic trends. A possible answer to this challenge may come from new integrated and technology-supported services [3].

The silver digital patient has already integrated eHealth applications and services in his/her everyday life.

The most important demands that a silver digital patient expects from eHealth tools and devices are the following:

- To have an elderly specific design for avoiding the digital-divide;

- To be adaptable to heterogeneous user-groups;

- To provide warns in case of critical status;

- To ensure data access in case of emergency;

- To facilitate a proactive management of health status;

- To support the receiving of accurate and reliable information about health status;

- To provide access to audio-visual tools that may help silver patients with poor eHealth literacy;

- To be able to integrate different eHealth applications to make them interoperable;

- To have the potential to strengthen the patient-physician communication;

- To allow patients to gather their own health data (using wearable technology) and send them to their physician;

- To have access 24h/7days access to services and information provided by eHealth tools.

\section{ProActive Ageing Platform}

ProActive Ageing Platform aims to contribute to a better social inclusion of silver patients and ageing people by providing services based on modern IT technology to facilitate and promote access to education and the labour market to this vulnerable group. Moreover, it provides practical, motivating, reliable and friendly informatics tools, dedicated to older patients, ageing persons, experts in the field of health, those who take care of ageing people, be they professionals or not, and business managers.

ProActive Ageing is under the development, the final version will be ready in 2017. 


\subsection{Specific objectives, characteristics and outcomes}

The specific objectives of the platform can be synthesized as follows:

- to promote access of silver patients and ageing persons to longlife learning and to ICT that will allow them to remain active, independent and fully involved in society;

- to implement an online centre for active ageing comprising person-centric tools, which represent the emerging solution that might tailor behaviouralmodification strategies to individual needs, and provide comprehensive active and healthy ageing awareness;

- to provide training and courses to healthcare staff and other types of carers, in order to increase the knowledge level and practical skills in the field of ageing people healthcare;

- to provide reliable anti-ageing knowledge, permanent updated with the help of an informatics platform that ensures a high degree of flexibility to changes and an effectiveness of the information management;

- to provide an integrated platform for online services that will establish an environment for the multidisciplinary collaboration among a variety of medical, biological and ICT specialties involved by the ageing domain.

The provided online services will increase the quality, efficiency and effectiveness of the measures imposed by the demographic changes that have to be taken for obtaining an active and healthy ageing. They are also envisaged to support the empowerment of the ageing persons regarding their own active and healthy life.

Due to the design focused on specific users and their needs, the online platform provides effective models of healthy and independent ageing, with real practical relevance, tailored to clearly identified beneficiaries, with less theoretical features.

ProActive Ageing can offer to ageing persons and silver digital patients a multidisciplinary, age-friendly tool in order to help them to have an active and healthy ageing. Healthcare professionals and other types of cares, on the other hand, will be offered an integrated tool for training courses and a toolkit designed for their researches, thus supporting them in the healthcare providing process. Lifelong learning, a person-centric approach and up-todate strategies, protocols and guides provided by ProActive Ageing are the key elements centralized in a new and feasible manner.

ProActive Ageing project involves distinct interdisciplinary research fields. This diversity enhances the team members' ability to bridge different scientific approaches and will further strengthen their research and development skills.

ProActive Ageing integrated platform for online services is structured into three thematic modules providing practical, engaging, accessible, motivating and customized tools that support longlife learning, with an accessible content for a large range of users and that are able to support social interaction and adaptability of individuals and of eHealth environment to the increasing ageing of the population:

- "Comprehensive learning courses for specialists taking care of ageing people" module: it offers innovative and practical training courses for specialist or non - specialist carers, including family, to improve and better understand their attributions in taking care of ageing persons;

- "Centre for Active Ageing" module: it provides online person-centric tools for a better knowledge and understanding of the ageing process, with lifelong learning facilities designed for ageing persons that want to remain active, healthy and independent;

- „A Toolkit to Support Medical Research Addressed to Healthy and Active Ageing" module: it provides centralized services for a better management of degenerative diseases, by providing instruments for specific innovative strategies/protocols/ algorithms, and knowledge exchange that will enhance the quality of researches and healthcare in the anti-ageing domain.

\section{2 “Centre for Active Ageing” module}

"Centre for Active Ageing" module represents a good example of a practical support that a silver digital patient can obtain from an 
eHealth application aiming to enhance his/her quality of life. It is designed to assist and facilitate people to know better their own ageing process and provides information on physical, psychological and social aspects of growing older.

"Centre for Active Ageing" module is developed as a web application consisting of a WordPress platform, running in an client/server architecture that uses open technologies of the Internet. WordPress websites in PHP run on a platform well known by the major search engines.

This makes them to be better indexed in the results that search engines return. The results compliance with current standards of this type of online platform are websites that load easily, have a consistent display in all web browsers and to whom update, maintenance and redesign of the dynamic website can be made with relative ease [9].

WordPress is the free and open-source content management system based on PHP and MySQL. It is installed on the web server that either is part of the Internet hosting service or is the network host itself. WordPress menu management has extended functionalities which can be modified to include the categories, the pages, being easy to operate [8].

The software infrastructure used for developing the model is L.A.M.P., consisting of:

- Linux - as a free, modern operating system that is in the vanguard of Web technologies;

- Apache - as a universal, independent portable Web server,;

- MySQL - as a database management system (relational DBMS);

- PHP - as a universal and independent programming language [5].
The combination of Linux, Apache, MySQL, and PHP, popular because of interaction, flexibility, customization, was chosen for the architecture of the module (see Figure 2).

The module is hierarchical structured into thematic components (see Figure 3):

\section{Home care}

a. Adjustments to the housing needs of the elderly;

b. How to employ a caretaker for an elderly person;

c. Types of home care services;

2. An active and independent person

d. Improvement of personal knowledge about healthy aging;

e. Aspects of an active and independent ageing;

f. Means of maintaining an active life;

g. Self-assessment tests;

3. Useful information

h. Emergency;

i. Legislation on seniors

This module provides accessible and relevant information aiming to support older people and their families in prolonging active and healthy lives and thereby increase the independence of elderly.

\subsection{Challenges for a successful implementation of ProActive Ageing}

In order to have a successful implementation, ProActive Ageing project has to carry out some challenges. Here are the most important ones and how ProActive Ageing responds to them:

1. Current insufficient attention to active and independent ageing:

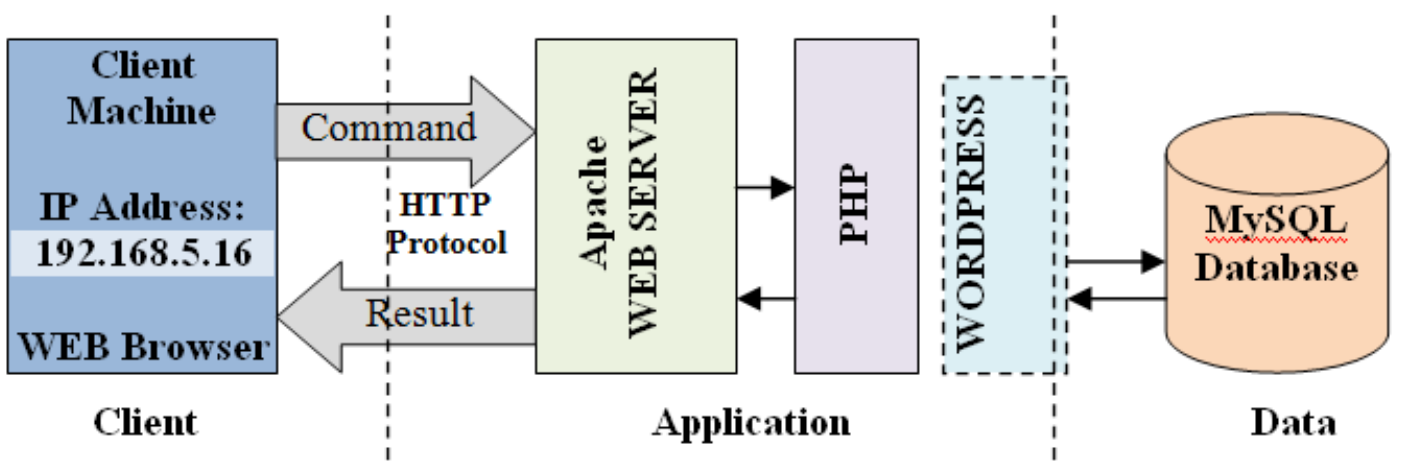

Figure 2. The architecture of "Centre for Active Ageing" module [9] 


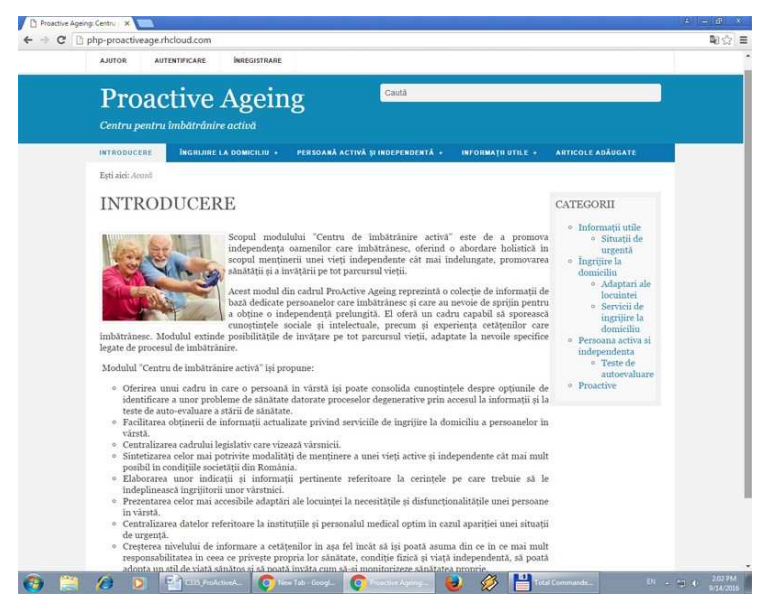

Figure 3. The main page of "Centre for Active Ageing" module

- The platform provides a functional model for a set of innovative, adapted online services that can support the active and independent living of silver digital patients and ageing people;

2. Risk of social marginalisation for ageing people:

- ProActive Ageing integrated platform for online services provides new directions, options and information able to facilitate social cohesion and an independent life for them;

3. The complexity and the volume of information of carers training needed:

- ProActive Ageing provides accessible, customized informatics tools supporting lifelong learning and content for a large range of users;

4. Low level of organised training for informal carers for ageing people:

- "Comprehensive learning courses for specialists taking care of ageing people" module provides means for lifelong learning, training and developing the skills of informal carers to update their skills and knowledge;

5. In some cases, ageing persons are not interested in and motivated to participate in learning, updating their knowledge or training in order to adapt to the agespecific changing:

- ProActive Ageing provides innovative methods of personal development, knowledge sharing and learning within the module "Centre for Active Ageing", adapted to the needs, diversity, and understanding of ageing persons;

6. Lack of awareness within health specialists regarding new strategies to tackle ageingrelated issues:

- "A Toolkit to Support Medical Research Addressed to Healthy and Active Ageing" module offers centralized services for a better management of degenerative diseases through providing different means enabling an integrated healthcare delivery;

7. Lack of relevant content for the ageingrelated problems:

- ProActive Ageing provides a reliable and updated scientific content due to its high level team members;

8. A lower confidence in using an online integrated platform, due to the inadequate skills, the lack of availability of, or access to, suitable training, missing health and ICT literacy, missing motivation or agerelated disabilities:

- ProActive Ageing provides tools and information tailored to the need of targeted users, namely ageing people, healthcare and social specialists and business managers. The user-centric design approach on which ProActive Ageing platform is based, offers a good understanding of user needs, correct translation of these into requirements, and always co-relate these to validation activities with real users in order to achieve effective and acceptable solutions and services;

9. Possible insufficient technical infrastructure or interoperability:

- ProActive Ageing is an open, interoperable and robust platform, independent from specialised hardware.

\section{Conclusions}

ProActive Ageing integrated platform for online services has considerable potential for enhancing the quality of life by providing new facilities and opportunities able to support mobility, independent and active living, and social participation. It facilitates the empowerment of the ageing persons by providing tools for a more "person-centric" social and healthcare system, in which individuals need to take an even more active role in personal care related decisions. 


\section{Acknowledgements}

"Prolonging Active Life for an Independent and Healthy Ageing" (ProActive Ageing) project is funded by the Romanian Partnership Program, Joint Applied Research Projects Subprogram under the National Plan for Research, Development and Innovation. The partnership is composed of "Dr. Victor Babeş" Clinical Hospital "Carol Davila" University of Medicine and Pharmacy, "Ana Aslan" National Institute of Gerontology and Geriatrics, National Institute for R\&D in Informatics and Siveco S.A.

\section{REFERENCES}

1. A BACKGROUND PAPER OF EUROPEAN COMMISSION, Growing the Silver Economy in Europe, 23 Feb 2015, Available at: http://ec.europa.eu/ research/innovation-union/pdf/activehealthy-ageing/ silvereco.pdf.

2. BIESDORF, S., F. NIEDERMANN, Healthcare's Digital Future, July 2014, Available at: http://www.mckinsey.com/ industries/healthcare-systems-and-services/ our-insights/healthcares-digital-future.

3. CAMARINHA-MATOS, L. M., H. AFSARMANESH, F. FERRADA, A. I. OLIVEIRA, J. ROSAS, A Comprehensive Research Roadmap for ICT and Ageing, Studies in Informatics and Control, ISSN 1220-1766, vol. 22(3), 2013, pp. 233-254.

4. EATOCK D., Briefing on the Silver Economy. Opportunities for Ageing, EPRS | European Parliamentary Research Service, July 2015, Available at: https://epthinktank.eu/2015/07/16/thesilver-economy-opportunities-from-ageing/

5. GERNER J., M. OWENS, E. NARAMORE, M. WARDEN, Professional LAMP: Linux, Apache, MySQL and PHP5 Web Development, 2013.
6. HEALTHY AGEING PROJECT, Healthy Ageing- a Challenge for Europe, (C) The Swedish National Institute of Public Health R 2006:29, ISSN: 1651-8624,ISBN: 917257-481-X, p. 16, Available at: http://ec.europa.eu/health/ph_projects/2003 /action1/docs/2003_1_26_frep_en.pdf

7. HIT - CONSULTANT CDW HEALTHCARE, Today's Digital Patient, Available at: http://www.cdwcommunit.com/ resources/infographic/digital-patient/

8. http://whichcmstochoose.com/wordpress.html

9. IANCULESCU M., A. ALEXANDRU, G. NEAGU, V. FLORAN, A. STANCIU, E. TUDORA, O. BICA, M. PIPEREA, Preliminary Development of "Centre for Active Ageing" Module, Research Report, Prolonging Active Life for an Independent and Healthy Ageing (ProActive Ageing) proj., 2015, pg. 23, 29, 35.

10. JOINT REPORT PREPARED BY THE EUROPEAN COMMISSION (DG ECFIN) AND THE ECONOMIC POLICY COMMITTEE (AWG), The 2012 Ageing Report; Economic and budgetary projections for the $27 \mathrm{EU}$ Member States (2010-2060); European Economy 2|2012, Available at: http://ec.europa.eu/economy finance/publications/european_economy $/ 20$ 12/pdf/ee-2012-2_en.pdf.

11. LEVINE D., S. LIPSITZ, J. LINDER, Trends in Seniors' Use of Digital Health Technology, 2011-2014, Journal of the American Medical Association, vol. 316(5), 2016, pp. 538-540.

12. SUDUC, A. M., M. BIZOI, F. G. FILIP, User Awareness about Information Systems Usability, Studies in Informatics and Control, vol. 19(2), 2010, pp. 145-152.

13. WORLD HEALTH ORGANIZATION, A contribution of the World Health Organization to the Second United Nations World Assembly on Ageing, Madrid, Spain, April 2002, p. 12. 